Silas Santos Carvalho ${ }^{1}$

Bruno Rodrigues de Oliveira ${ }^{2}$

Eliana Alves de Sá3

\section{Estratégias e ações no pré-natal para sifflis congênita: revisão de literatura}

RESUMO| Introdução: A sífilis é uma infecção sexualmente transmissível que tem cura e em qualquer estágio gestacional ou em qualquer estágio clínico da doença materna a transmissão vertical do Treponema pallidum pode ocorrer e acarretar em graves consequências. Objetivo: Analisar criticamente, com base na literatura, as estratégias e as ações para prevenção e controle da sífilis congênita durante o pré-natal. Métodos: Revisão integrativa da literatura, incluindo artigos em idioma português, publicados entre 2010 e 2018, através de busca nas bases de dados eletrônicas SciELO (Scientific Eletronic Library Online), LILACS (Literatura Latino-Americana e do Caribe em Ciências da Saúde) e BDENF (Base de Dados de Enfermagem). Foi encontrado um total de 112 estudos, dos quais 11 foram selecionados e consultados integralmente. Resultados: Foi possível identificar que as oportunidades para a tomada de ações e estratégias para a prevenção da sífilis congênita têm sido perdidas, apontando falhas na assistência pré-natal em todos os níveis de atenção à saúde. Conclusão: As ações de prevenção e controle da sífilis congênita incluem acesso ao pré-natal de qualidade, integral e humanizado e o rastreamento sorológico, com início imediato do tratamento das gestantes identificadas e seus parceiros. Todavia, faz-se necessária a realização de qualificação dos profissionais de saúde e educação em saúde para a população.

Palavras-chave | Sífilis; Gestantes; Sífilis Congênita; Controle; Cuidado pré-natal; Saúde da mulher.

${ }^{1}$ Universidade Estadual de Feira de Santana. Feira de Santana/BA, Brasil.

${ }^{2}$ Universidade Salvador. Feira de Santana/BA, Brasil.

${ }^{3}$ Faculdade Ateneu. Feira de Santana/BA, Brasil. 


\section{INTRODUÇÃO|}

A sífilis congênita (SC) consiste em uma disseminação hematogênica do agente Treponema pallidum ( $\mathrm{T}$. pallidum) de uma gestante infectada, seja ela não tratada ou tratada inadequadamente para o seu concepto via transplacentária ${ }^{1-4}$. Em qualquer estágio gestacional ou clínico da doença materna pode ocorrer a transmissão vertical do treponema, sendo que os principais fatores que apontam a probabilidade de transmissão são: o estágio da sífilis na gestante e a duração que o feto sofreu no meio intrauterino ${ }^{1}$.

Dentre os fatores de risco para a aquisição de sífilis por uma mulher em idade fértil, destacam-se: baixo nível socioeconômico, promiscuidade sexual, inacessibilidade ao sistema de saúde, uso de drogas e escolaridade. No que se refere à SC, acrescentam-se como fatores de risco ausência de assistência pré-natal e gestante adolescente e/ou sem parceiro fixo. O fator de risco mais importante para a SC é o acompanhamento gestacional inadequado no pré-natal ${ }^{5,6}$.

De acordo com o Boletim Epidemiológico do Ministério da Saúde ${ }^{7}$, nos últimos 10 anos no Brasil, a taxa de SC teve um aumento progressivo, sendo que no ano de 2007 , a taxa era de 1,9 casos/1.000 nascidos vivos e, em 2017, essa taxa aumentou mais de quatro vezes, passando para 8,6 casos / 1.000 nascidos vivos.

$\mathrm{Na}$ Bahia, os casos notificados de SC em menores de 1 ano de idade apontaram, em 2015, o número de 1.166 casos (taxa de 5,6 casos/1.000 nascidos vivos); em 2016, 1.388 casos (taxa de 6,7 casos/1.000 nascidos vivos) e, em 2017, número de 473 casos notificados e taxa de $7,82^{\circ}{ }^{8}$. No município de Feira de Santana-BA, foi notificado um total de 644 casos de sífilis congênita em menores de 1 ano nos últimos 20 anos (1998 a 2018), e, em 2017, a taxa foi de 6,8 casos/1.000 nascidos vivos ${ }^{9}$.

Para o devido controle da SC, são necessárias estratégias que incluem a garantia de que todas as gestantes recebam assistência pré-natal e que, em sua rotina, a triagem da sífilis ocorra a todas as mulheres e seus parceiros; a realização do exame VDRL na primeira consulta, no início do terceiro trimestre e no parto; a disponibilidade do tratamento a todas as gestantes infectadas e seus parceiros e a devida notificação dos casos de sífilis materna e congênita à vigilância epidemiológica ${ }^{1}$.
A Atenção Básica deve priorizar o diagnóstico das gestantes e parceiros com sífilis. Portanto, é necessário que os profissionais de saúde estejam aptos para identificar e classificar as manifestações clínicas e os estágios da sífilis, bem como interpretar os resultados, pois estes são imprescindíveis para o controle da doença, permitindo o diagnóstico e a resposta terapêutica ${ }^{2}$.

Embora a SC seja uma doença de fácil prevenção e baixo custo, ainda consiste em um grande desafio para as políticas públicas, vistos os dados publicados pelo Ministério da Saúde, em 2017, no Boletim Epidemiológico da Sífilis, mostrando aumento significativo de casos em gestantes.

Diante do exposto, é relevante a compreensão sobre as estratégias e as ações para um diagnóstico e tratamento imediato e adequado da sífilis na gestação a fim de prevenila por meio do acesso ao pré-natal.

Nesse contexto, o objetivo deste estudo é analisar criticamente, com base na literatura, as estratégias e as ações para prevenção e controle da SC durante o pré-natal.

\section{MÉTODOS |}

Este estudo constitui-se de uma revisão integrativa da literatura, a fim de produzir um consolidado a partir das publicações científicas sobre as ações e estratégias para o enfrentamento da SC no pré-natal.

A coleta de dados foi realizada no período de 08 a 12 de abril de 2019, mediante os descritores: "sífilis", "sífilis congênita", "sífilis na gestação" e, para tanto, utilizou-se a base de dados Scientific Eletronic Library Online (SciELO), Literatura Latino-Americana e do Caribe em Ciências da Saúde (LILACS) e Base de Dados de Enfermagem (BDENF). As listas de referência dos artigos selecionados foram checadas para identificar outros artigos relevantes.

Os critérios de inclusão foram artigos que discorressem sobre a temática proposta, publicados no idioma português entre os anos de 2010 e 2018 e que se encontravam disponíveis na íntegra e gratuitamente. Foram excluídos os resumos, as teses, as monografias, as dissertações, os estudos de caso e aqueles com duplicidade entre as bases de dados. 
Após a busca, foi encontrado um total de 112 artigos, 24 artigos pré-selecionados que, após submetidos aos critérios de inclusão e exclusão, foram reduzidos a 11 publicações que visaram responder à questão norteadora do estudo.

Os dados foram analisados com base nas etapas dos princípios de Minayo ${ }^{10}$. Na primeira, foi realizada uma pré-análise dos artigos selecionados, identificando suas hipóteses e objetivos a fim de triar quais seriam utilizados na etapa posterior. $\mathrm{Na}$ segunda etapa, o material foi explorado e foram identificadas as ideias propostas nos artigos para a sua compreensão. Por fim, os artigos foram discutidos e interpretados de acordo com o objetivo proposto.

A revisão foi organizada e sistematizada a partir da categoria que emergiu com maior força e relevância do nível empírico: "Prevenção e controle da sífilis no pré-natal".

\section{RESULTADOS/DISCUSSÃO |}

Foram identificados, inicialmente, com base nos descritores, 112 trabalhos. Destes, apenas 24 estudos foram encontrados disponíveis em textos completos gratuitamente em meio eletrônico e com os critérios de inclusão estabelecidos, sendo excluídos da presente revisão os trabalhos incompletos, duplicados e que não tinham a temática como seu foco principal. Restaram, então, 11 artigos consultados integralmente. Em relação às bases de dados de indexação dos estudos, oito estavam indexados na SciELO, um na LILACS e dois na BDENF.

No Quadro 1 é possível observar uma breve descrição dos 11 estudos que compuseram a amostra para análise e discussão dos resultados.

Diante dos estudos analisados, observa-se que as oportunidades para a tomada de ações e estratégias para a prevenção da SC têm sido perdidas, apontando falhas na assistência pré-natal em todos os níveis de atenção à saúde, especialmente na Atenção Básica.

Essas falhas no pré-natal são evidenciadas na cobertura ineficiente que dificulta o acesso ao pré-natal, no tratamento inadequado das gestantes e nos parceiros sexuais. Somandose a isso, é visível a falta de preparo e conhecimento dos profissionais para a tomada de decisão oportuna frente à gestante com sífilis.
É importante a realização do teste sorológico em todas as gestantes na primeira consulta de pré-natal no primeiro trimestre de gestação, devendo repetir a sorologia no início do terceiro trimestre para que a terapêutica necessária seja aplicada em tempo hábil ${ }^{11}$.

Quando a assistência pré-natal é deficiente, ocorrem falhas no tratamento da gestante com sífilis e, consequentemente, resulta em um aumento no número de casos de SC. Além disso, o tratamento e o diagnóstico da SC envolvem a realização de exames, podendo gerar danos familiares e individuais e custos adicionais ao sistema de saúde, por isso, o enfrentamento da SC é mais complexo que o da sífilis materna ${ }^{12}$.

Estudos $^{13,14}$ evidenciaram que a dificuldade do desuso de preservativos, principalmente em relações estáveis, é uma dificuldade a ser considerada, então deve haver uma postura dos profissionais de saúde que favoreça o acolhimento e negociações, juntamente com a paciente, de estratégias com o parceiro a fim de não ocorrer a reinfecção da sífilis.

Em estudo ${ }^{15}$, verificou-se a ocorrência de falhas no acesso e na realização de exames e obtenção do diagnóstico da sífilis, que ocorriam tardiamente, na ocasião de internação, evidenciando uma rotina de pré-natal inadequada e oportunidades de controle da doença perdidas.

Observa-se que os parceiros não são tratados ou iniciam o tratamento e o abandonam e, somado a isso, há uma falta de conhecimento sobre a importância de sua realização ${ }^{11,16}$. O tratamento dos parceiros sexuais possibilita a quebra do ciclo de transmissão da sífilis. Em concordância, pesquisa ${ }^{17}$ reforça que a gestante com sífilis deve passar por um diagnóstico e tratamento adequados, juntamente com o seu parceiro sexual, evitando agravos.

Investigação $^{18}$ refere que a assistência pré-natal de qualidade com a atenção correta e precoce à gestante, inclusão de ações de promoção à saúde, orientação sexual e reprodutiva, realização de exames preconizados para o período gestacional é essencial para prevenção de agravos ao concepto. Todavia, a quantidade de consultas realizadas no pré-natal não é a garantia de um controle efetivo da sífilis ${ }^{19}$. Isso fica evidente em estudo ${ }^{18}$ com gestantes que iniciaram as consultas no primeiro trimestre e ainda houve persistência de um diagnóstico tardio. 
Quadro 1 - Características dos artigos do levantamento bibliográfico

\begin{tabular}{|c|c|c|}
\hline AUTOR / ANO & OBJETIVO & MÉTODO \\
\hline $\begin{array}{l}\text { Padovani C, Oliveira RR, } \\
\text { Pelloso SM, } 2018\end{array}$ & $\begin{array}{l}\text { Analisar a prevalência de sífilis na gestação e sua } \\
\text { associação com características socioeconômicas, } \\
\text { histórico reprodutivo, assistência no pré-natal e no } \\
\text { parto e características do recém-nascido. }\end{array}$ & $\begin{array}{l}\text { Estudo retrospectivo, transversal, realizado } \\
\text { a partir das notificações de sífilis gestacio- } \\
\text { nal e sífilis congênita. }\end{array}$ \\
\hline $\begin{array}{l}\text { Lazarini FM, Barbosa DA, } \\
2017\end{array}$ & $\begin{array}{c}\text { Avaliar a eficiência da intervenção educacional no } \\
\text { conhecimento dos profissionais de saúde da Aten- } \\
\text { ção Básica e verificar o impacto nas taxas de trans- } \\
\text { missão vertical da sífilis congênita }\end{array}$ & $\begin{array}{l}\text { Estudo quase-experimental, conduzido na } \\
\text { cidade de Londrina, Paraná, no período en- } \\
\text { tre } 2013 \text { e } 2015 \text {. }\end{array}$ \\
\hline $\begin{array}{l}\text { Nunes JT, Marinho ACV, } \\
\text { Davim RMB, Silva GGO, } \\
\text { Félix RS, Martino MMF, } \\
2017\end{array}$ & $\begin{array}{l}\text { Discutir as ações do enfermeiro na atenção pré-na- } \\
\text { tal a gestante com sífilis e identificar dificuldades } \\
\text { encontradas pelos profissionais na adesão ao tra- } \\
\text { tamento das gestantes e parceiros. }\end{array}$ & $\begin{array}{c}\text { Estudo qualitativo, tipo descritivo-explora- } \\
\text { tório, desenvolvido com } 04 \text { mulheres na } \\
\text { faixa etária de } 40 \text { a } 55 \text { anos, através de } \\
\text { entrevistas semiestruturadas. }\end{array}$ \\
\hline $\begin{array}{l}\text { Lafetá KRG, Martelli Júnior } \\
\text { H, Silveira MF, Paranaíba } \\
\text { LMR, } 2016\end{array}$ & $\begin{array}{l}\text { Identificar e descrever casos de sífilis congênita e } \\
\text { materna notificados e não notificados em uma cida- } \\
\text { de de médio porte. }\end{array}$ & $\begin{array}{c}\text { Estudo descritivo e retrospectivo para ava- } \\
\text { liar } 214 \text { prontuários de gestantes e recém- } \\
\text { nascidos. }\end{array}$ \\
\hline $\begin{array}{l}\text { Suto CSS, Silva DL, Almei- } \\
\text { da ES, Costa LEL, Evange- } \\
\text { lista TJ, } 2016 .\end{array}$ & $\begin{array}{l}\text { Caracterizar a assistência prestada à gestante com } \\
\text { diagnóstico de sífilis durante o pré-natal em unida- } \\
\text { des de saúde da família. }\end{array}$ & $\begin{array}{l}\text { Estudo transversal por meio da análise } \\
\text { de relatórios de sistemas de informação e } \\
\text { questionários estruturados sobre exposi- } \\
\text { ção à sífilis em gestante. }\end{array}$ \\
\hline $\begin{array}{l}\text { Araújo MAL, Barros VL, } \\
\text { Moura HJ, Rocha AFB, } \\
\text { Guanabara MAO, } 2014\end{array}$ & $\begin{array}{l}\text { Analisar a estrutura e o processo de trabalho dos } \\
\text { profissionais para desenvolver ações de prevenção } \\
\text { e controle da SC. }\end{array}$ & $\begin{array}{l}\text { Pesquisa de campo em } 89 \text { unidades primá- } \\
\text { rias. }\end{array}$ \\
\hline $\begin{array}{l}\text { Magalhães DMS, Kawagu- } \\
\text { chi IAL, Dias A, Calderon } \\
\text { IMP, } 2013\end{array}$ & $\begin{array}{l}\text { Estabelecer o perfil das gestantes com VDRL re- } \\
\text { agente acompanhadas em maternidades públicas } \\
\text { do DF, Brasil, dos recém-nascidos de mães com } \\
\text { sífilis que apresentaram sinais clínicos da doença } \\
\text { congênita e verificar a conduta clínica de acordo } \\
\text { com as normas preconizadas pelo Ministério da } \\
\text { Saúde. }\end{array}$ & $\begin{array}{l}\text { Estudo descritivo, com } 67 \text { gestantes e } \\
\text { puérperas notificadas no Sistema Nacional } \\
\text { de Notificação de Agravos para coleta de } \\
\text { informações sociodemográficas e relacio- } \\
\text { nadas ao diagnóstico e tratamento no Dis- } \\
\text { trito Federal, Brasil, entre } 2009 \text { e } 2010 \text {. }\end{array}$ \\
\hline $\begin{array}{l}\text { Campos ALA, Araújo MAL, } \\
\text { Melo SP, Andrade RFV, } \\
\text { Gonçalves MLC, } 2012\end{array}$ & $\begin{array}{l}\text { Analisar o perfil sociodemográfico e comportamen- } \\
\text { tal dos parceiros sexuais, a proporção daqueles } \\
\text { inadequadamente tratados e os motivos para o não } \\
\text { tratamento. }\end{array}$ & $\begin{array}{l}\text { Estudo transversal quantitativo em } 05 \text { ma- } \\
\text { ternidades públicas de Fortaleza, Ceará. }\end{array}$ \\
\hline $\begin{array}{l}\text { Saraceni V, Miranda AE, } \\
2012\end{array}$ & $\begin{array}{l}\text { Investigar a relação entre a cobertura da Estraté- } \\
\text { gia de Saúde da Família nas regiões brasileiras e } \\
\text { a razão de casos de sífilis em gestante e de sífilis } \\
\text { congênita observados entre os casos estimados, } \\
\text { para o ano de } 2008 .\end{array}$ & $\begin{array}{l}\text { Estudo de dois agravos, através da obser- } \\
\text { vação dos casos obtidos no SINNANNET, } \\
\text { disponível na página da SVS/MS, por ma- } \\
\text { crorregião do Brasil para o ano de } 2008 \text {. }\end{array}$ \\
\hline $\begin{array}{l}\text { Nascimento MI, Cunha AA, } \\
\text { Guimarães EV, Alvarez FS, } \\
\text { Oliveira SRSM, Villas Bôas } \\
\text { EL, } 2011\end{array}$ & $\begin{array}{l}\text { Descrever as características de gestações compli- } \\
\text { cadas por sífilis materna e óbito fetal. }\end{array}$ & $\begin{array}{l}\text { Estudo retrospectivo descritivo com revisão } \\
\text { de } 48 \text { prontuários de gestantes com sífilis e } \\
\text { desfecho de óbito fetal, entre } 2005 \text { e } 2008 .\end{array}$ \\
\hline $\begin{array}{l}\text { Campos ALA, Araújo MAL, } \\
\text { Melo SP, Gonçalves MLC, } \\
2010\end{array}$ & $\begin{array}{l}\text { Verificar o percentual de gestantes com exame } \\
\text { VDRL reagente em qualquer período gestacional, } \\
\text { ou na ocasião do parto, que não foram adequada- } \\
\text { mente tratadas, buscando identificar os motivos da } \\
\text { não adequação do tratamento e apresentar o perfil } \\
\text { sociodemográfico, obstétrico e epidemiológico des- } \\
\text { sas gestantes. }\end{array}$ & $\begin{array}{l}\text { Estudo transversal e descritivo, em } 05 \text { ma- } \\
\text { ternidades públicas em Fortaleza, Ceará, } \\
\text { Brasil. }\end{array}$ \\
\hline
\end{tabular}

Fonte: SciELO, LILACS e BDENF. 
Durante as consultas de pré-natal o profissional deverá orientar a gestante, o parceiro e demais familiares a respeito da importância dos cuidados quando ocorre a sorologia positiva para sífilis, bem como o tratamento e medidas adequadas ${ }^{20}$. O teste rápido (TR) em parceiros de gestantes é muito importante e um ganho para o controle da sífilis ${ }^{21}$.

Estudo ${ }^{15}$ corrobora afirmando que o controle da doença é comprometido à medida que as pacientes não recebem um tratamento e acompanhamento no pré-natal. O uso do TR para a sífilis é apropriado como estratégia para agilizar a abordagem da sífilis nas primeiras visitas.

A periodicidade da realização do exame VDRL de forma não preconizada pelo MS permite refletir que essa questão faz com que a assistência no pré-natal ocorra de forma que favoreça o risco para o aparecimento de SC no recémnascido $^{19}$.

Assim, a SC está inserida no quadro de causa perinatal evitável, podendo ser controlada mediante diagnóstico e tratamento adequados no período gestacional ${ }^{14}$. A Estratégia de Saúde da Família, sendo a base de assistência primária no Brasil, precisa aliar as tecnologias existentes a uma assistência de qualidade para a redução da $\mathrm{SC}^{22}$.

Aproximadamente dois terços dos nascidos vivos com SC são assintomáticos, e nos demais as características clínicas são relacionadas à classificação da doença $a^{23}$. Por outro lado, a morte fetal, morte neonatal, prematuridade, baixo peso ao nascer e/ou a infecção congênita são agravos da sífilis gestacional não tratada ou tratada de forma inadequada ${ }^{11}$.

Outro fator relacionado à qualidade da assistência no pré-natal é a falta de informações no cartão da gestante, pois o seu correto preenchimento é fundamental para dar seguimento ao tratamento da sífilis na gestante e para que haja uma redução significante da sífilis nesse período. Para que ocorra a diminuição e o controle da SC, é necessário o comprometimento dos gestores e profissionais de saúde com a qualidade dos serviços prestados no pré-natal, considerando a importância dos registros referentes à gestante $^{19}$.

A notificação compulsória é outra medida que tem o objetivo de diminuir e eliminar a SC, pois fornece dados para que se possa realizar uma análise que leve a intervenções para a redução de sua incidência e das suas consequências ${ }^{17}$. Todavia, evidencia-se na atual conjuntura uma subnotificação, o que sinaliza a ausência de política para o controle da sífilis e deficiência na qualidade da assistência $^{18,21}$.

A falta temporária do medicamento nas unidades básicas de saúde configurou-se um problema identificado para o devido tratamento, assim como grande resistência das gestantes com relatos de ser muito doloroso, justificando o abandono do tratamento. Reforça-se a necessidade de a gestante e seu parceiro realizarem o tratamento em conjunto e de modo adequado ${ }^{21}$.

A vigilância ativa à saúde das gestantes dá embasamento às ações que visam à eliminação da SC por meio do rastreamento e tratamento em todos os níveis de atenção. A educação em saúde é um fator primordial para favorecer a competência ao sujeito para compreender e executar as orientações preconizadas ${ }^{20}$.

$\mathrm{O}$ enfermeiro tem um papel importante no processo da abordagem às gestantes com sífilis e no controle da sífilis gestacional e, dentre outras ações, destacam-se: a informação ao tratamento de forma adequada; uso de preservativos nas relações sexuais; educação em saúde; prática de exames e captação dos parceiros que não aderem ao tratamento ${ }^{23}$.

Pesquisa $^{24}$ evidenciou falta de realização de atividades educativas, bem como um percentual baixo de profissionais capacitados para o manejo da sífilis. Embora haja disponibilidade de tratamento simples e de baixo custo, a compreensão do diagnóstico possui complexidade e pode dificultar o manejo por parte dos profissionais. Portanto, a educação permanente desses profissionais que atuam no pré-natal deve ser realizada em intervalos regulares e com supervisão continuada.

\section{CONCLUSÃO |}

Diante dessa revisão de literatura, pode-se inferir que a SC é passível de prevenção, uma vez que a gestante infectada seja identificada precocemente e tratada de forma adequada. O diagnóstico e o tratamento preconizados são de baixo custo, porém a Organização Mundial de Saúde classifica a SC como um importante problema de saúde pública a fim de pôr em prática as políticas públicas de saúde voltadas para o seu controle e criar utras mais eficientes. 
O panorama atual dos estudos sobre SC evidencia que as ações de prevenção e controle da doença estão pautadas em medidas preconizadas pelo MS, que incluem acesso ao prénatal de qualidade, integral e humanizado, e o rastreamento sorológico, com início imediato do tratamento das gestantes identificadas e seus parceiros.

Importante assinalar a necessidade da realização de qualificação para os profissionais de saúde em todos os níveis de atenção, bem como a educação em saúde para a população acerca da relevância do controle da sífilis e seus agravos.

Diante do exposto, faz-se necessária a produção de mais estudos sobre a SC quanto à prevenção e seu controle, visto que o enfoque maior é na epidemiologia e nos seus aspectos clínicos.

\section{REFERÊNCIAS |}

1. Brasil. Ministério da Saúde. Secretaria de Vigilância em Saúde. Programa nacional de DST e Aids. Protocolo para a prevenção de transmissão vertical de HIV e sífilis: manual de bolso. Brasília: Ministério da Saúde; 2007.

2. Ministério da Saúde. Sífilis. Boletim Epidemiológico. 2018; 49(45):1-48.

3. Feitosa JAS, Rocha CHRR, Costa FS. Artigo de revisão: sífilis congênita. Rev Med Saúde Brasília. 2016; 5(2):286-97.

4. Cardoso ARP, Araújo, MAL, Cavalcante MS, Frota MA, Melo SP. Análise dos casos de sífilis gestacional e congênita nos anos de 2009 a 2010 em Fortaleza, Ceará, Brasil. Ciênc Saúde Coletiva. 2018; 23(2):563-74.

5. Guinsberg R, Santos AMN. Critérios diagnósticos e tratamento da sífilis congênita. São Paulo: Sociedade Brasileira de Pediatria; 2010.

6. Magalhães DMS, Kawaguchi IAL, Dias A, Paranhos Calderon IM. Sífilis na gestação e sua influência na morbimortalidade materno-infantil. Comun Ciênc Saúde. 2011; 22 (sup. esp. 1):43-54.

7. Brasil. Ministério da Saúde. Secretaria de Vigilância em Saúde. Protocolo clínico e diretrizes terapêuticas para a prevenção da transmissão vertical do HIV, sífilis e hepatites virais. Brasília: Ministério da Saúde; 2018.

8. Ministério da Saúde. Sífilis. Boletim Epidemiológico. 2017; 48(36):1-44.

9. Brasil. Ministério da Saúde [Internet]. Indicadores e dados básicos da sífilis nos municípios brasileiros [acesso em 21 jan 2019]. Disponível em: URL: http://indicadoressifilis. aids.gov.br.

10. Minayo MCS. O desafio do conhecimento: pesquisa qualitativa em saúde. São Paulo: Hucitec; Abrasco, 2010.

11. Padovani C, Oliveira RR, Pelloso SM. Sífilis na gestação: associação das características maternas e perinatais em região do sul do Brasil. Rev Latino-Am. Enfermagem. 2018; 26.

12. Magalhães DMS, Kawaguchi IAL, Dias A, Calderon IMP. Sífilis materna e congênita: ainda um desafio. Cad Saúde Pública. 2013; 29(6):1109-20.

13. Campos ALA, Araújo MAL, Melo SP, Andrade RFV, Gonçalves MLC. Sífilis em parturientes: aspectos relacionados ao parceiro sexual. Rev Bras Ginecol Obstet. 2012; 34(9):397-402.

14. Domingues RMSM, Saraceni V, Hartz ZMA, Leal MC. Sífilis congênita: evento sentinela na qualidade da assistência pré-natal. Rev Saúde Pública. 2013; 47(1):147-57.

15. Nascimento MI, Cunha AA, Guimarães EV, Alvarez FS, Oliveira SRSM, Villas Bôas EL. Gestações complicadas por sífilis materna e óbito fetal. Rev Bras Ginecol Obstet. 2011; 34(2):56-63.

16. Sonda EC, Richter FF, Boschetti G, Casasola MP, Krumel CF, Machado CPH. Sífilis congênita: uma revisão da literatura. Rev Epidemiol Control Infect. 2013; 3(1):2830 .

17. Suto CSS, Silva DL, Almeida ES, Costa LEL, Evangelista TJ. Assistência pré-natal a gestante com diagnóstico de sífilis. Rev Enferm Atenção Saúde. 2016; 5(2):18-33.

18. Lafetá KRG, Martelli Júnior H, Silveira MF, Paranaíba LMR. Sífilis materna e congênita, subnotificação e difícil controle. Rev Bras Epidemiol. 2016; 19(1):63-74. 
19. Campos ALA, Araújo MAL, Melo SP, Gonçalves MLC. Epidemiologia da sífilis gestacional em fortaleza, Ceará, Brasil: um agravo sem controle. Cad Saúde Pública. 2010; 26(9):1747-55.

20. Lazarini FM, Barbosa DA. Intervenção educacional na Atenção Básica para a prevenção da sífilis congênita. Rev Latino-Am. Enferm. 2017; 25:e2845.

21. Nunes JT, Marinho ACV, Davim RMB, Silva GGO, Félix RS, Martino MMF. Sífilis na gestação: perspectivas e condutas do enfermeiro. Rev Enferm UFPE on line. 2017; 11(12):4875-84.

22. Saraceni V, Miranda AE. Relação entre a cobertura da Estratégia de Saúde da família e o diagnóstico de sífilis na gestação e sífilis congênita. Cad Saúde Pública. 2012; 28(3):490-6.

23. Brasil. Ministério da Saúde. Secretaria de Vigilância em Saúde. Diretrizes para controle da sífilis congênita: manual de bolso. Brasília: Ministério da Saúde; 2006.

24. Araújo MAL, Barros VL, Moura HJ, Rocha AFB, Guanabara MAO. Prevenção da sífilis congênita em Fortaleza, Ceará: uma avaliação de estrutura e processo. Cad Saúde Coletiva. 2014; 22(3):300-06.

Correspondência para/Reprint request to:

\section{Silas Santos Carvalho}

Universidade Estadual de Feira de Santana,

Av. Transnordestina,

Novo Horizonte, Feira de Santana/BA, Brasil

E-mail:ssc.academico@hotmail.com

Recebido em: 29/05/2019

Aceito em: 13/08/2020 